\title{
La Méditerranée, un trésor pédagogique
}

\section{Filhol, Benoît}

Universidad Católica San Antonio Murcia, bfilhol@ucam.edu

\begin{abstract}
Resumen
El Mediterráneo, "mar blanco » o mare nostrum constituye un tema de estudio literario con un gran potencial pedagógico en atención a la herencia cultural y a los valores que ha legado y a los vínculos tejidos con sus habitantes, un auténtico tesoro que debemos explotar en clase de Francés como Lengua Extranjera (FLE) o en francés para sus nativos. Para ello, con esta publicación se propone un nuevo enfoque que permita el estudio de la literatura a través de proyectos (unidades de acción) tal como lo recomienda el MCER y algunos currículos de educación secundaria europeos. Se trata de contribuir a cubrir el vacío metodológico sobre el tema señalado por varios especialistas en Didáctica de FLE. El objetivo del proyecto puede revestir varias formas pero, para nosotros, debe provenir de un oficio del mundo literario (escritor, editor, comisario de exposición, etc.) para que la clase realice una acción que sea lo más cercana posible a la de un actor real. El ejemplo que ofrecemos aquí y que podrá servir de modelo para otros proyectos se organiza en cuatro etapas compuestas de micro-tareas unidas las unas a las otras. Los estudiantes guiados por el profesor descubrirán una práctica profesional, se sumergirán en el tema del Mediterráneo y sin perder de vista la problemática adoptada construirán una exposición que presentarán durante la inauguración de la misma.
\end{abstract}

Palabras clave : Mediterráneo ; Literatura ; Pedagogía de proyectos ; Enfoque orientado a la acción; Exposición.

\section{Résumé}

La Méditerranée, "mer blanche » ou mare nostrum, constitue, au regard de l'héritage culturel et des valeurs qu'elle a légués et des liens tissés avec ses habitants, un thème d'étude littéraire au grand potentiel pédagogique, un authentique trésor que nous nous devons d'exploiter en classe de FLE ou de FLM. Pour le faire, nous proposons, au travers de cette publication, une nouvelle approche qui permet l'étude de la littérature par projets (unités d'action) comme le préconisent le CECRL et certains programmes d'éducation secondaire européens. Il s'agit là de contribuer à combler le vide méthodologique sur le sujet signalé par plusieurs didacticiens du FLE. Évidemment, notre proposition didactique n'élude pas tous les travaux réalisés jusque-là sur la pédagogie de projet mais essaie d'adapter cette dernière et la perspective actionnelle à l'étude de la littérature. L'objectif du projet peut revêtir plusieurs formes mais doit, selon nous, provenir d'un métier du monde littéraire (auteur, éditeur, commissaire d'exposition, etc.) pour que la classe réalise une action qui soit le plus proche possible de celle de l'acteur réel. L'exemple que nous offrons ici et qui pourra servir de modèle pour d'autres projets s'organise en quatre étapes composées de micro-tâches toutes reliées les unes aux autres. Les apprenants-usagers, guidés par le professeur sont amenés à découvrir une pratique professionnelle, se plongent dans le thème de la Méditerranée et, sans perdre de vue la problématique adoptée, construisent une exposition qu'ils présentent lors d'un vernissage.

Mots-clés : Méditerranée ; littérature ; pédagogie de projet ; perspective actionnelle ; exposition.

\footnotetext{
Abstract

The Mediterranean Sea, «white sea» or mare nostrum, constitutes a literary subject to study with a strong teaching potential concerning its cultural heritage and the values that it bequeaths plus the bonds with its inhabitants; this is an authentic treasure worth considering in the subject of French as a Foreign Language (FFL) as well as in French for native speakers. To this purpose, my piece of research introduces a new approach to a project-based literature teaching in consonance with CEFR and some secondary education
} 
curriculums in Europe. We would like to contribute to fulfill some methodological gaps in this area which have previously been highlighted by some FFL educational specialists. This teaching proposal does not skip previous works about Project-Based Learning, but tries to adapt the latest methodology and an actionoriented approach to literature studies. In our view, the main focus of the project should be real life jobs related to literature (writer, editor, exhibition curator, etc.). In this way, students can perform actions as closed as possible to those real actors do. The example shown in this paper, which could be used as a model for other projects, is divided into four stages including micro-tasks which are coupled together. Guided by the teacher, learners will discover a job practice; they will plunge into the subject of the Mediterranean Sea; and without losing sight of the current issue, they will prepare an exhibition that will be presented during its inauguration.

Keywords : Mediterranean Sea; Literature; Project-Based Learning; Action-oriented approach; Exhibition.

\section{Introduction}

L'enseignement de la littérature en Français Langue Maternelle et en Français Langue Étrangère a connu plusieurs périodes de remise en question et de débats. Au cours des dix dernières années, plusieurs hommes de lettres, parmi eux Tzvetan Todorov (2007), Antoine Compagnon (2007), Jean-Marie Schaeffer (2011), Vincent Jouve (2010) ont évoqué une crise du littéraire et de son apprentissage. Dans leurs essais, ces différents critiques ont tous émis des diagnostics personnels mais ont aussi tous été d'accord pour remettre en cause la manière scolaire d'étudier le texte littéraire et ont défendu son importance dans le cadre d'une formation humaniste et démocratique. Dans le domaine du Français Langue Étrangère, on ne peut pas dire que le littéraire ait bénéficié et jouisse actuellement d'un traitement de faveur. Depuis l'apparition de l'approche communicative, ce sont les textes authentiques qui sont sur le devant de la scène au détriment de leurs homologues littéraires. Toutefois, dans un élan d'optimisme toujours nécessaire à l'innovation didactique, nous préférons parler, quant à nous, plutôt que de crise, de moment d'évolution ou de changement. En effet, plusieurs configurations didactiques ont émergé et continuent d'apparaître avec comme objectif de redorer le blason de la littérature pour l’École du Vingt-unième siècle.

Parmi ces possibilités, la pédagogie de projet semble avoir un temps d'avance. Elle est déjà pratiquée en France et en Espagne par de nombreux professeurs mais force est de constater qu'elle ne s'appuie pas encore sur un cadre théorique qui la légitime et la consolide en envisageant une articulation précise entre pédagogie de projet et littérature. Nous souhaitons donc contribuer à cette réflexion, dans un premier temps, en examinant les attentes du travail par projets pour le littéraire, puis en proposant un exemple de projet, qui s'inspire de la thématique du XXV ${ }^{\text {ème }}$ colloque AFUE puisque nous présenterons un projet sur la Méditerranée, qui constitue, à nos yeux, un trésor pédagogique.

\section{L’enseignement de la littérature au travers de la pédagogie de projet}

La pédagogie de projet, comme nous le signalions dans l'introduction, semble s'imposer pour l'étude du littéraire grâce au plus qu'elle apporte par rapport aux méthodes pratiquées jusqu'à présent. Tout d'abord, elle permet d'aller au-delà de certaines configurations didactiques où le texte littéraire est utilisé exclusivement pour illustrer un point grammatical par des exemples ou comme document-source d'un aspect culturel.

Toutefois, si elle permet de faire évoluer l'enseignement de la littérature en classe de FLE vers une expérience d'enseignement-apprentissage plus complète, elle ne constitue pas une rupture totale, compte tenu du fait qu'elle n'est pas incompatible avec toutes les activités réalisées jusqu'à maintenant en classe de littérature. Nous faisons référence à la lecture à voix haute, à la confrontation de documents de genres et de modes distincts, à l'exploitation grammaticale, au travail sur un questionnaire qui évalue la compréhension globale et la compréhension spécifique, à la production écrite et orale, etc.

Le vrai plus de la pédagogie de projet réside dans le fait qu'elle insuffle de l'action au cœur de l'enseignement. C'est en cela qu'elle se rapproche de la configuration didactique préconisée, depuis 2001, par le Cadre Européen Commun de Référence pour les Langues. Comme s'est efforcé de montrer Christian Puren depuis 2001, la donne a bien changé avec 
l'apparition du texte du Conseil de l'Europe et, avec la perspective actionnelle, il ne s'agit plus de préparer les apprenantsusagers à communiquer efficacement en langue étrangère mais de leur enseigner à « partager avec les autres les mêmes conceptions de l'action commune » (Puren, 2006a : 40). Christian Puren est aussi un des premiers didacticiens à avoir pensé cette articulation entre l'enseignement-apprentissage de la littérature et la conception de projets et le moins qu'on puisse dire est qu'il a présagé à la pédagogie de projet un bel avenir. Nous citons à nouveau Puren qui dans un autre article de 2006 écrivait : « on peut concevoir des projets sociaux réels plus originaux faisant appel à la littérature, la limite étant celle de l’imagination de l'enseignant, des opportunités de terrain et de la motivation de ses élèves » (2006b).

Avec cette méthodologie, nous sommes amenés à travailler à partir de périodes didactiques ou séquences d’apprentissageaction pour reprendre la terminologie de Claire Bourguignon qui ne se basent plus seulement sur une situation de communication simulée, comme c'était le cas avec l'approche communicative, mais autour d'actions collectives authentiques que les apprenants-usagers doivent préparer et réaliser en groupes.

Pour l'instant, il n'existe que très peu de travaux envisageant l'articulation entre littérature et pédagogie de projet. Nous devons donc nous pencher sur la bibliographie existant sur la pédagogie de projet pour déterminer une possible application de cette dernière au champ littéraire.

Le pédagogue suisse Philippe Perrenoud a produit une réflexion profonde sur l'utilisation du projet dans l'enseignement. Dans la lignée de William Heard Kilpatrick, un des pères de la pédagogie de projet, il définit un projet comme « une initiative collective gérée par le groupe classe qui débouche sur une production concrète » (Perrenoud, 1999). Pour cela, les élèves s’impliquent dans plusieurs tâches, se confrontent à des savoirs et à des savoir-faire liés à la gestion du projet. Perrenoud propose dix attentes que peut viser un projet d'enseignement et qui sont particulièrement intéressantes pour la conduite d'un projet littéraire. Nous retiendrons, pour cet article, les trois premières attentes mentionnées par l'auteur et nous nous efforcerons de montrer en quoi la pédagogie de projet peut être bénéfique pour l'enseignement de la littérature.

Tout d'abord, comme signale Perrenoud, le travail par projets sert à « entraîner la mobilisation de savoirs et savoir-faire acquis, construire des compétences » (1999). La confrontation à des " problèmes littéraires » doit permettre le transfert et la mobilisation de savoirs qui habituellement sont abordés séparément. Autrement dit, tant que l’apprenant-usager n’est pas impliqué dans une situation similaire à celle d'un projet réel, on peut douter du fait qu'il soit capable de conjuguer toutes les compétences nécessaires pour résoudre une question posée. Deuxièmement, toujours selon le pédagogue suisse, le projet permet de donner à voir des pratiques sociales qui accroissent le sens des savoirs et des apprentissages scolaires » (Perrenoud, 1999). Le travail en classe se rapproche des situations que nous pouvons rencontrer dans la vie réelle, ce qui contribue à ajouter plus de sens aux notions, méthodes et connaissances. L'apprenant-usager a l'impression de réaliser quelque chose de concret et utile, donc sa motivation augmente. Le thème de la motivation prend encore plus de sens quand on se situe dans une discipline comme la littérature qui est trop souvent considérée comme abstraite et que l'on met un peu de côté dans un monde qui privilégie les logiques économique, scientifique et technologique. La troisième attente est en relation directe avec la précédente. Pour Perrenoud, le projet « permet de découvrir de nouveaux savoirs, de nouveaux mondes, dans une perspective de sensibilisation ou de motivation » (1999). Les apprenants-usagers se retrouvent dans des contextes sociaux réels qui leur ouvrent la porte de nombreux secteurs professionnels et culturels. Ces incursions dans des disciplines connexes permettent une transversalité et la construction d'une culture littéraire et générale essentielle pour comprendre la complexité du monde actuel.

Les sept autres attentes sont plus générales et ainsi directement transférables au travail du littéraire au travers de la pédagogie de projet. Pour cela, nous n’avons pas jugé nécessaire de les commenter de manière détaillée dans cette étude. Toutefois, ce qui en ressort est que le travail au travers de projets littéraires développe des compétences qui vont au-delà de la compétence littéraire. Et c'est bien là que réside à nos yeux un autre grand intérêt de l'application de la pédagogie de projet à la littérature. Le projet permet de mobiliser des compétences qu’Isabelle Bordalo et Jean-Paul Ginestet (1993) identifient comme «des compétences clefs» ou «transversales». Parmi elles, se trouvent "problématiser», « rechercher », « se documenter », « réaliser », « organiser », " planifier », " contrôler », « critiquer », " prendre en compte », etc. Toutes ces compétences exigent des outils linguistiques et des savoir-faire qui ne sont pas exclusifs du domaine littéraire et que l'étudiant nécessitera et exploitera dans d’autres contextes.

Il est légitime et souhaitable que ce soit, comme que l'a souligné Christian Puren (2006b), l'imagination et la liberté pédagogique du professeur qui animent l'élaboration et le développement de cette méthodologie mais nous pensons qu'une manière productive est de l'envisager en se basant sur les métiers liés à la réalité littéraire -ceux de l’édition, de 
l'écriture, de la recherche et de la sphère culturelle- pour construire l'apprentissage au travers de pratiques réelles. Nous insistons : les possibilités sont certes infinies mais nous souhaiterions offrir quelques propositions de projets littéraires qui pourraient aider le professeur :

Tableau 1. Quelques exemples de projets envisagés à partir de la pratique réelle

\begin{tabular}{ll}
\hline \multicolumn{1}{c}{ Acteur littéraire } & \multicolumn{1}{c}{ Objectif du projet } \\
\hline Professeur-Chercheur & Rechercher un thème et publier une étude \\
Editeur & $\begin{array}{l}\text { Éditer un texte et le publier (sur internet, centre } \\
\text { éducatif, format papier, etc.), participer à un conseil } \\
\text { éditorial pour décider quels textes publier, etc. } \\
\text { Écrire une critique et la publier, présenter une } \\
\text { Journaliste/critique }\end{array}$ \\
$\begin{array}{l}\text { cuvre } \\
\text { Correcteur de style }\end{array}$ & $\begin{array}{l}\text { Corriger un texte en vue d'une future publication } \\
\text { Chercher une maison d'édition à un écrivain qui }\end{array}$ \\
sont littéraire & souhaite publier une ouvre. \\
Écrivain(e) & Créer une œuvre et la publier \\
Conservateur/Commissaire & Organiser une exposition et réaliser le vernissage \\
\hline
\end{tabular}

Dans le cadre d'un projet littéraire, le texte n'est plus le seul objectif comme dans l'enseignement traditionnel de la littérature mais un moyen pour exécuter une tâche sociale. Dans cette logique, l'action n'est plus au service des textes mais ce sont les textes et autres documents qui sont au service de l'action. Les apprenants-usagers ne sont plus de simples lecteurs mais des auteurs et surtout des acteurs. Comme cela se produit dans la perspective actionnelle, on fera en sorte de réaliser les actions proposées en dehors de la classe, si c'est possible, et sinon, au moins, on les exécutera de la manière la plus réaliste possible. Enfin, comme l’on peut l’observer dans les exemples de projets proposés, la littérature n'est plus mise à l'écart comme ce fut le cas avec les approches didactiques qui ont précédé la perspective actionnelle mais fait partie intégrante de l'enseignement-apprentissage dans un processus holistique et en interrelation.

\section{Un exemple de projet littéraire : une exposition sur la Méditerranée}

Dans cette démarche de projet, il convient, comme nous l'avons signalé, de donner du sens aux apprentissages. Or, pour cela, nous sommes convaincus que le projet lui-même doit avoir du sens. On se demandera donc -et le lecteur se demandera sans doute- pourquoi aborder le thème de la Méditerranée ? Quel sens cela a ? Que peut-il apporter à un processus d'apprentissage de Français Langue Étrangère ? En quoi la Méditerranée constitue un trésor pédagogique ? Nous allons tenter de répondre à ces questions en donnant plusieurs éléments de réponse.

Premièrement, la Méditerranée est, pour les apprenants, un thème familier. Ils ont tous des connaissances sur le sujet, ont tous un rapport proche ou lointain avec cet espace. On peut donc partir des pré-acquis -et il faudra le faire-pour construire de nouveaux savoirs. Familier et surtout attractif car pour eux, ce thème est synonyme de soleil, de vacances, de baignades; il évoque des souvenirs qu'ils se feront un plaisir de se remémorer pendant le projet.

Il nous semble, par ailleurs, que la Méditerranée est un thème idéal pour travailler à l'acquisition de l'éducation humaniste, la Bildung pour reprendre le terme allemand. Composée de connaissances, de compétences et de valeurs, la culture humaniste ou Bildung se définit comme le développement personnel de potentialités intellectuelles, affectives et éthiques de l’être humain au sein de la collectivité à laquelle il appartient.

En effet, la Méditerranée n’est pas seulement une mer. C'est un « espace matriciel, une machine à faire de la civilisation » (cité par Liauzu, 2002 : 66), selon les mots de Paul Valéry, un espace géographique qui a vu naître la Démocratie et les civilisations, Grecque et Romaine, qui ont elles-mêmes engendré, entre autres, les peuples d’Occident. 
Or, à partir du $20^{\text {ème }}$ siècle, pour beaucoup de penseurs et d'écrivains -Edgar Morin et Paul Morand, pour ne citer qu'eux, la Méditerranée a perdu de sa superbe et il n’est pas démesuré de dire, à la lumière de leurs écrits, qu’on ne voit plus, qu’on ne reconnaît plus cette Méditerranée aujourd’hui. Pour Edgar Morin, par exemple, rares sont ceux qui écoutent de nos jours cette Méditerranée, berceau de nos civilisations. La voix de ces intellectuels -que nous pourrons invoquer dans notre projet- nous invite donc à prendre de la hauteur pour examiner cette mer autrement et à se demander ce qu'il reste aujourd'hui de l'image d'une mer accueillante et enrichissante.

Sans tomber dans une vision idéalisatrice du passé et dans un catastrophisme du présent, il convient donc de s’interroger sur les problématiques qui agitent la Méditerranée à l’heure actuelle. Il est intéressant en effet d’opérer un va-et-vient entre passé et présent pour comparer et mettre en perspective des réalités menacées, en décadence ou disparues. Les sauts chronologiques et les anachronismes provoqués et volontaires qui seront à l'œuvre au cœur du projet sont nécessaires à l'heure où le symbolique recule au profit de produits culturels prêts à consommer et superficiels. Par conséquent, la tension entre passé, présent et futur devra apparaître et être au cœur de la problématique de notre projet.

La Méditerranée est un thème littéraire par excellence. Pour certains, elle est l'espace du texte, pour d'autres c'est le lieu qui a vu naître la poésie ou même " une mer littéraire » (cité par Siegfried, 1944 : 44). Une chose est sûre : les écrivains sont justement ceux qui, depuis plusieurs siècles, ont pensé et continuent d’interroger cette Méditerranée. Nous nous rendrons vite compte -et ce sera un des objectifs du projet que nous allons énoncer- que les mythes méditerranéens originels sont toujours convoqués par les auteurs contemporains car, malgré le temps qui s’est écoulé, ils sont encore d'actualité, ont encore des choses à nous dire et à nous apprendre.

Enfin, la Méditerranée est un outil pédagogique idéal pour travailler l'interculturel et le pluriculturel. Depuis les phéniciens, la « mer au milieu des terres » a été un espace d'échange et une terre d'accueil. Aujourd'hui encore, le long de ces côtes, sa population est faite d’un mélange cosmopolite. Ce creuset se retrouve évidemment dans les salles de classes. Au-delà de la prise en compte de la réalité multiculturelle de la Méditerranée de la part du professeur lors de la planification du projet, les élèves auront à cœur d’évoquer leur culture d’origine. Confrontés à des savoirs, des savoirfaire et savoir-être différents, ils seront donc amenés à aller au-delà d'une simple compréhension mutuelle et à travailler ce que Christian Puren nomme la compétence co-culturelle qui consiste à «élaborer et mettre en œuvre une culture d'action commune dans le sens d’un ensemble cohérent de conceptions partagées » (2007).

Pour donner un exemple et expliquer le déroulement d'un projet pédagogique, nous avons choisi celui d'une exposition sur la Méditerranée. Quant à la manière d’organiser l'apprentissage, nous nous sommes inspirés des travaux de Claire Bourguignon et de son concept de scénario d'action-apprentissage. En effet, cette démarche ou processus d’apprentissage, qui mêle langue et culture, et que Bourguignon définit comme «une simulation basée sur une série de tâches communicatives, toutes reliées les unes aux autres, visant l'accomplissement d'une mission plus ou moins complexe par rapport à un objectif » (2007) nous paraît parfaitement adéquate pour structurer notre projet. Pour cela, nous avons également respecté un des principes proposé par Bourguignon pour qui « chaque micro-tâche est imbriquée dans l'autre et au service de l'accomplissement de la mission » (2007). Pour garantir cette imbrication, nous avons opté pour quatre phases composées de micro-tâches conduisant à la tâche finale où se matérialise le projet.

Voici comment pourrait s’articuler notre projet à partir de cette esquisse qui prétend couvrir plusieurs séances pour un niveau correspondant à celui d'un utilisateur indépendant de la langue ${ }^{1}$ (niveau B2 selon le Cadre Européen Commun de Référence pour les Langues Étrangères) :

1) La première phase est une phase d'ancrage avec comme objectif l'évaluation des pré-acquis. Le professeur montre plusieurs images très diverses ayant pour cadre la Méditerranée (par exemple, le Colisée de Rome, une vue du ciel de Benidorm, une table méditerranéenne ou un olivier) et la classe réalise une activité de remue-méninges dans laquelle les apprenants-usagers doivent répondre à la question suivante : Quelle Méditerranée êtes-vous ? Cela permet, d’autre part, aux étudiants de faire des hypothèses et de parler de la situation passée, actuelle et future de cet espace géographique, le professeur les orientant dans cette direction.

2) La deuxième phase « en contexte » permet de rentrer dans le vif du sujet et vise l'accomplissement de deux objectifs. Tout d'abord, il s'agit pour les apprenants-usagers de découvrir et analyser la pratique d'une exposition culturelle. Le

\footnotetext{
${ }^{1}$ Toutefois, le projet est adaptable à d'autres niveaux en fonction des documents choisis et surtout du niveau des tâches et des activités proposées.
} 
professeur leur propose donc un exemple d'exposition sur le thème de la Méditerranée, par exemple un document visuel, sonore ou audio-visuel sur l'exposition « Méditerranées » organisée à Marseille en 2013. S’ensuit une réflexion sur la préparation et la construction d'une exposition. Les étudiants sont invités notamment à répondre aux questions suivantes : Est-ce qu'une exposition est simplement un inventaire ou catalogue de documents ? Qu'est-ce qui permet de donner une unité à l'ensemble ? Cette phase d'immersion permet aussi aux apprenants-usagers de confronter, par petits groupes, les contenus mentionnés dans l'étape antérieure avec de nouvelles données pour une construire une problématique qui constituera le fil conducteur de l'exposition qu’ils devront réaliser. Pour les aider à formuler cette problématique, ils peuvent s'appuyer sur deux documents : un fragment de « Penser la Méditerranée et méditerranéiser la pensée », article d’Edgar Morin et un extrait du documentaire Méditerranée, notre mer à tous de Yann Arthus-Bertrand. La problématique devra refléter cette tension entre héritage, présent et avenir de la Méditerranée.

3) La troisième phase est une étape d'exploration et de recueil d'informations et de ressources, effectuée en binômes ou par petits groupes. Les apprenants sont amenés à analyser des mythes et des thèmes méditerranéens qui sont autant d'outils pour répondre à la problématique formulée dans la phase précédente. Ces informations serviront de ressources pour l'exposition en fin de séquence. Le professeur propose aux apprenants trois extraits sur le thème de l'olivier, «L'arbre qui traverse les temps » : un de l'Odyssée lorsqu’Ulysse fait naufrage chez les Phéaciens, un de Manosque-des-Plateaux de Jean Giono («Quand j'étais tout petit, je jouais, puis j'avais faim » (Giono, 1998 : 68)) et le poème « L'olivier amer » de Mehmet Yashin. Ensuite, le professeur réoriente la réflexion sur la question, déjà entrevue, du tourisme en Méditerranée à partir de l'étude d'un passage du roman de Julien Blanc-Gras Touriste. Finalement, pour se projeter sur le futur de la Méditerranée, les étudiants sont invités à réfléchir sur le drame humanitaire qui secoue actuellement la Méditerranée avec l'immigration massive de personnes en provenance d'Afrique et du Proche-Orient. Pour cela, ils sont amenés à mettre en parallèle un extrait du roman de Laurent Gaudé Eldorado (2006) avec un fragment d'un reportage radiophonique sur le même thème. Des activités portant spécifiquement sur la langue peuvent compléter cette étape, toujours dans l'optique de fournir aux étudiants les ressources nécessaires pour mener à bien la mission qui leur a été confiée. Le professeur peut également demander aux apprenants d’approfondir en leur suggérant de rechercher et d'analyser d'autres mythes ou thèmes qu'ils associent à la Méditerranée.

4) À présent, les apprenants-usagers disposent de ressources suffisantes pour passer à la phase de réalisation du projet (tâche finale). Ils dressent une liste de tâches à réaliser, se mettent d'accord pour le partage du travail et passent à l'action. Tout au long de cette étape, le professeur guide les étudiants en les invitant à sélectionner, organiser et présenter les documents et matériaux choisis pour l'exposition. Les différentes sections de celle-ci peuvent être ensuite montées dans la salle de classe ou dans un espace de l'établissement prévu à cet effet, à moins que la classe ait décidé d'élaborer un poster multimédia à l'aide d'une Technologie de l'Information et de la Communication (TIC), comme par exemple Glogster. Les apprenants-usagers doivent également mettre en place le vernissage en préparant la présentation de l'exposition et en faisant la liste des invités. Le jour du vernissage, les apprenants jouent le rôle des membres de l’organisation et présentent les différentes sections de l'exposition.

\section{Conclusion}

En guise de conclusion, nous souhaiterions rappeler les avantages du travail du littéraire au travers de la réalisation de projet en espérant avoir pu les illustrer au travers de l'exemple proposé. La pédagogie de projet insuffle de l'action à l'enseignement de la littérature et motive les apprenants pour l'apprentissage d’une discipline quelque peu délaissé depuis un certain temps dans l'enseignement-apprentissage des langues étrangères. Cette approche permet l'acquisition de nombreuses compétences qui vont au-delà du littéraire sans sacrifier ce dernier. En effet, à nos yeux, elle ne délaisse pas la compétence littéraire, mais au contraire, elle favorise pleinement son apprentissage en tenant compte de sa dimension affective et imaginative mais aussi de sa composante théorique et pratique. 


\section{Références bibliographiques}

Bordalo, Isabelle et GinESTET, Jean-Paul (1993). Pour une pédagogie du projet. Paris : Hachette.

BouRguignON, Claire (2007). Apprendre et enseigner les langues dans la perspective actionnelle : le scénario d'apprentissage-action. Association des Professeurs de Langues Vivantes. http://www.aplv-languesmodernes.org/spip.php?article865 [Consulté le 29 juin 2016].

Compagnon, Antoine (2007). La littérature, pour quoi faire? Paris : Bayard.

Giono, Jean (1998). Manosque-des-Plateaux. Paris : Folio, Gallimard.

Jouve, Vincent (2005). Pourquoi étudier la littérature. Paris : Armand Colin.

Liauzu, Claude (2002). « Le migrant méditerranéen, toujours suspect » dans Confluences Méditerranée, n 42, p. 65-70.

Perrenoud, Philippe (1999). Apprendre à l'école à travers des projets : pourquoi? Comment? Université de Genève. <http://www.unige.ch/fapse/SSE/teachers/perrenoud/php_main/php_1999/1999_17.html> [Consulté le 29 juin 2016].

PuREN, Christian (2006a). « De l'approche communicative à la perspective actionnelle » dans Le Français dans le monde, n³47, p. 37-40.

- (2006b). Explication de textes et perspective actionnelle : la littérature entre le dire scolaire et le faire social. Association des

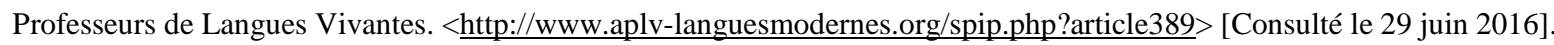

- (2007). Perspectives actionnelles et perspectives culturelles en didactique des langues-cultures : vers une perspective co-actionnelle co-culturelle. Association des Professeurs de Langues Vivantes. <http://www.aplv-languesmodernes.org/spip.php?article844> [Consulté le 29 juin 2016].

SCHAeffer, Jean-Marie (2011). Petite écologie des études littéraires. Pourquoi et comment étudier la littérature. Paris : Thierry Marchaisse.

SIEGFRIED, André (1944). Vue générale de la Méditerranée. Paris : NRF, Gallimard.

Todorov, Tzvetan. (2007). La littérature en péril. Paris : Flammarion. 